\title{
Relação entre aleitamento materno e hábitos de sucção não nutritivos
}

\author{
The relation between maternal breast feeding \\ and non-nutritive sucking habits
}

\author{
Suzely Adas Saliba Moimaz ${ }^{1}$ \\ Najara Barbosa Rocha ${ }^{1}$ \\ Artênio José Isper Garbin ${ }^{1}$ \\ Orlando Saliba ${ }^{1}$
}

${ }^{1}$ Faculdade de Odontologia de Araçatuba, Universidade Estadual Paulista. Rua José Bonifácio 1193. 16015-050 Araçatuba SP. sasaliba@foa.unesp.br
Abstract This study sought to identify the type and duration of breastfeeding received by children and establish the association with non-nutritive suction habits. A retrospective, transversal study was conducted using a questionnaire given to 330 mothers or persons responsible for 3 to 6-year-old children registered in kindergartens in Araçatuba, State of São Paulo, after obtaining their free and informed consent. It was revealed that $86.4 \%$ of mothers breastfed their children. With respect to duration, only $33.4 \%$ of these mothers breastfed exclusively after 6 months. The average time of exclusive breastfeeding received by children was 3.84 months and complementary breastfeeding was 11.68 months. Of this total, $53.3 \%$ reported that their infants manifested non-nutritive suction habits and of these children, $70.45 \%$ were not exclusively breastfed for the first 6 months, there being a significant association between both $(p<=0.05)$. Pacifier use was the most frequent habit $(44.4 \%)$, showing a statistically significant association with breastfeeding time $(p<0.0001)$. It was concluded that breastfeeding was practiced, albeit for a lesser duration than considered indispensable for the baby's development, there being an association between duration and type of breastfeeding and non-nutritive suction habits.

Key words Breast feeding, Maternal breastfeeding, Non-nutritive suction habits
Resumo Este estudo objetivou identificar o tipo e o período de tempo de aleitamento recebido por crianças e verificar a associação com hábitos de sucção não nutritivos. Trata-se de um estudo transversal, retrospectivo, no qual foi aplicado um questionário em 330 mães ou responsáveis por crianças de 3 a 6 anos matriculadas em pré-escolas de Araçatuba (SP), após consentimento livre e esclarecido. Observou-se que $86,4 \%$ das mães amamentaram seus filhos. Quanto ao período de tempo, apenas 33,4\% delas amamentaram exclusivamente no peito após seis meses de vida. O tempo médio de amamentação materna exclusiva recebida foi 3,84 meses e o de aleitamento complementar foi de 11,68 meses. Do total, 53,3\% relataram que os filhos apresentavam hábitos de sucção não nutritivos, e destes 70,45\% não foram amamentados exclusivamente no peito durante os seis primeiros meses de vida, existindo associação significativa entre os dois $(p \leq 0,05)$. O uso de chupeta foi o hábito mais frequente (44,4\%), apresentando associação estatisticamente significante com o tempo de aleitamento $(p<0,0001)$. Conclui-se que o aleitamento materno foi praticado, porém, por período de tempo inferior ao período considerado indispensável ao desenvolvimento do bebê, existindo associação entre tempo e tipo do aleitamento e hábitos de sucção não nutritivos.

Palavras-chave Aleitamento materno, Amamentação, Hábitos de sucção não nutritivos 


\section{Introdução}

A prática do aleitamento materno no Brasil está longe do cumprimento da recomendação da Organização Mundial da Saúde (OMS) ${ }^{1}$ e do Ministério da Saúde ${ }^{2}$, ou seja, amamentação exclusiva até o sexto mês de vida e a continuidade do aleitamento materno complementar até o segundo ano de vida ou mais.

No Brasil, foi verificado que mesmo que a maioria (por volta de $92 \%$ ) das mulheres inicie a amamentação natural de seus filhos, mais da metade das crianças já não se encontram em amamentação exclusiva no primeiro mês de vida ${ }^{3}$.

A garantia da saúde materno-infantil é uma das metas para este milênio, e entre uma das suas estratégias de promoção da saúde destaca-se o incentivo à prática do aleitamento materno. Desde anos 80, a OMS e o Fundo das Nações Unidas para a Infância (Unicef) direcionaram esforços para o incentivo de uma política voltada para a prática do aleitamento materno, incluindo diversos documentos que mostram a importância do aleitamento materno e sua prática ${ }^{4}$.

$\mathrm{O}$ avanço tecnológico e a entrada das mulheres no mercado de trabalho, associados a forte publicidade sobre a comodidade e a praticidade do leite industrializado, constituíram a base do declínio do aleitamento materno $0^{5-8}$.

As vantagens do leite materno incluem o seu conteúdo nutricional, melhor capacidade de absorção interna, previne alergias e problemas respiratórios, propicia desenvolvimento psicológico mais favorável, melhores defesas imunológicas, exerce um papel importante na redução da mortalidade infantil, além dos aspectos afetivos entre a mãe e o bebề, ${ }^{90}$. $\mathrm{O}$ aleitamento natural traz ainda benefícios para a nutriz. A prática da amamentação no seio promove melhor involução genital no período pós-parto, diminui a incidência do câncer mamário e útero, além dos aspectos da praticidade em sua manipulação, não resultando em gastos financeiros para a família ${ }^{11}$.

A amamentação natural é importante para o crescimento normal da criança ${ }^{12}$, representa o fator inicial do bom desenvolvimento dentofacial, favorecendo a obtenção de uma oclusão dentária normal e, consequentemente, uma mastigação correta no futuro. Estimula também o crescimento anteroposterior da mandíbula. Além disso, determina uma relação adequada entre estruturas duras e moles do aparelho estomatognático, permitindo tonicidade e postura correta da língua, com lábios em perfeito vedamento, propiciando o estabelecimento da respiração nasal. Assim, promove um bom desenvolvimento das estruturas orais envolvidas no ato de sugar, contribuindo para uma boa fonoarticulação, mastigação, deglutição e respiraçãoo $0^{9,11,13,14}$. $\mathrm{O}$ aleitamento artificial não supre essas necessidades que o aleitamento natural supre ${ }^{6}$.

A relação entre forma de aleitamento e hábitos de sucção não nutritivos tem sido descrita por vários autores ${ }^{7,9}$. Os hábitos se instalam com maior frequência em crianças que não tiveram amamentação natural, pois o impulso neural da sucção está presente desde a vida intrauterina e é normal na criança, garantido sua sobrevivência. O aleitamento natural, além de alimentar o bebê, tem a função de satisfazer a sucção, devido à ação dos músculos exercidos durante a mamada ${ }^{15}$. Quando a criança é aleitada por mamadeiras, o fluxo de leite é bem maior que a amamentação natural, portanto a criança se satisfaz nutricionalmente em menor tempo e com menor esforço. O prazer emocional com relação ao impulso da sucção não é atingido, e a criança procura por isso substitutos como dedo, chupeta e objetos para satisfazer-se ${ }^{16}$.

Geralmente, os hábitos bucais nocivos instalam-se com maior frequência em quem não obteve amamentação natural, para tentar suprir o impulso da sucção, pois ela satisfaz, além da necessidade de alimento, importantes necessidades psicológicas ${ }^{17}$.

O dano causado ao sistema estomatognático pelo hábito depende das variáveis: frequência, intensidade e duração do hábito (Tríade de Graber). Todo hábito que perdurar após os três anos ou tiver alta frequência será mais deletério e capaz de causar oclusopatias graves ${ }^{18}$.

Dentre os hábitos bucais deletérios, podemse destacar: sucção do polegar e outros; sucção e mordida dos lábios; deglutição atípica; postura; onicofagia; sucção habitual do lápis; chupetas e outros objetos e perturbações funcionais gnatológicas: abrasão, bruxismo diurno e noturno, respirador bucal ${ }^{14}$.

Baseado na necessidade da realização de mais estudos que comprovem a relação desmame e hábitos, estabelecendo relações entre práticas de aleitamento para evitar os danos à saúde, este estudo objetivou identificar o tipo e o período de tempo de aleitamento em crianças com até seis anos de idade; verificar os hábitos de sucção não nutritivos e sua relação com o aleitamento. O projeto foi aprovado pelo Comitê de Ética em Pesquisa com Seres Humanos da Faculdade de Odontologia de Araçatuba, da Universidade Estadual Paulista. 


\section{Metodologia}

A população de estudo foi constituída por crianças com 3 a 6 anos de idade, de ambos os sexos, matriculadas em escolas municipais de educação infantil da cidade de Araçatuba (SP). O dimensionamento da amostra foi efetuado levando-se em consideração o nível de mensuração nominal, população finita, nível de confiança de $5 \%$ e erro amostral admitido de $5 \%{ }^{19}$. O tamanho da amostra foi de 330 escolares entre 3 e 6 anos.

Esta pesquisa foi do tipo descritiva, transversal, na qual foram entrevistadas 330 mães empregando-se um questionário, previamente testado em estudo piloto, contendo questões sobre as condições socioeconômicas e outras variáveis como: aleitamento, hábitos presentes nas crianças, respiração bucal e doenças na infância. As entrevistas foram realizadas por uma única pessoa, previamente calibrada, após consentimento livre e esclarecido dos participantes da pesquisa, na entrada ou saída das crianças nas escolas e nas reuniões de pais.

Os dados foram analisados pelo Programa EpiInfo 2000 e pacote estatístico GraphPad ${ }^{20}$. Foram utilizados os testes Qui-quadrado $\left(\chi^{2}\right)$ e Teste Exato de Fischer, ao nível de significância de $5 \%(\mathrm{p} \leq 0,05)$.

\section{Resultados e discussão}

O aleitamento materno é considerado fundamental para a promoção e a proteção da saúde das crianças em todo o mundo. É praticado por grande parte da população mundial, porém ainda está distante da situação desejada para se garantirem melhores níveis nutricionais das crianças e consequentemente diminuir a mortalidade infantil em todo o mundo.

No levantamento realizado no Brasil, em 1996, sobre a situação do aleitamento materno, a mediana da amamentação exclusiva foi de um mês, variando conforme o nível educacional das mães. Ao final do primeiro ano, apenas $40,8 \%$ das crianças continuavam sendo amamentadas ${ }^{3}$. Esses dados ficam distante dos ideais recomendados pela $\mathrm{OMS}^{1}$ e pelo Ministério da Saúde ${ }^{2}$, que recomendam amamentação materna exclusiva durante seis meses e aleitamento complementar até pelo menos dois anos de idade ou mais.

Nesta pesquisa, foi observado que $86,4 \%$ (285) das mães amamentaram seus filhos no peito, sendo semelhantes aos dados de Bezerra et al. ${ }^{21}$. Porém, dessas mães, a grande maioria
$(66,6 \%)$ não amamentou exclusivamente seus filhos (Gráfico 1). Este período é o tempo recomendado pelas instituições nacionais ${ }^{2}$ e internacionais $^{22,23}$, ou seja, pelo menos seis meses.

O tempo médio em meses de aleitamento materno exclusivo recebido pelas crianças da amostra foi 3,84, e o período de aleitamento complementar foi de 11,68.

Baldrighi et al. ${ }^{6}$ observaram dados semelhantes com o estudo de 180 crianças de 4 a 6 anos, encontrando valores como $73,33 \%$ de crianças não amamentadas exclusivamente por período suficiente. Sousa et al. ${ }^{16}$ avaliaram 126 crianças de 2 a 6 anos e verificaram que 93,6\% mamaram no peito, porém $63,97 \%$ não foram amamentadas por pelo menos seis meses. Já Vasconcelos et al. ${ }^{24}$ acompanharam 852 crianças até 24 meses, sendo a taxa de aleitamento materno exclusivo de 24 dias apenas.

Bittencourt et al. ${ }^{7}$ relataram em seu estudo, com 239 pais ou responsáveis pelas crianças de 4 a 6 anos em fase pré-escolar da cidade do Rio de Janeiro (RJ), que $82 \%$ foram amamentadas naturalmente, mas exclusivamente com leite materno por apenas $18 \%$ das mães.

Os valores obtidos neste estudo $(33,4 \%$ em regime exclusivo até seis meses e $62,1 \%$ amamentadas até 12 meses) foram superiores àqueles encontrados no estudo nacional realizado em 1999, no qual apenas 9,7\% com leite natural exclusivo aos seis meses e $44,2 \%$ das crianças eram amamentadas, como forma complementar, até os 12 meses $^{3}$.

Em relação ao principal motivo de desmame (Gráfico 2), as mães responderam como motivos: trabalho da mãe $(20,9 \%)$; falta de leite $(19,4 \%)$; não aceitação da criança $(18,2 \%)$; filho

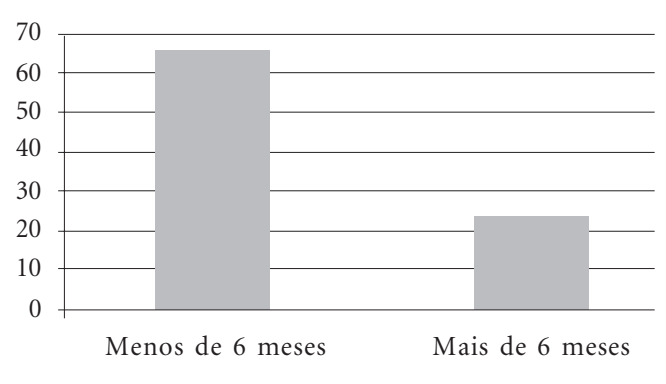

Aleitamento materno exclusivo

Gráfico 1. Distribuição percentual das 330 crianças segundo o período de tempo de aleitamento natural exclusivo recebido - Araçatuba (SP). 
já crescido (13,8\%); outros $(6,8)$; doença da mãe $(6,2 \%)$; leite fraco $(4,9 \%)$; gravidez $(2,8 \%)$; dor ao amamentar $(4,6 \%)$ e doença da criança $(2,5 \%)$. Ferreira e Toledo ${ }^{9}$ Baldrighi et al. ${ }^{6}$ relataram que um dos fatores contribuintes para o desmame precoce é a entrada das mulheres no mercado de trabalho, mesmo sendo permitido por leis trabalhistas amamentar seus filhos durante o trabalho, protegendo assim esse ato.

Foi observado que as mães ou responsáveis pelas crianças tinham renda familiar considerada baixa $(70,6 \%$ recebiam menos que dois salários mínimos), só que contraditoriamente, mesmo sabendo que o leite materno é gratuito e uma forma de alimentação sem nenhum gasto, portanto uma forma de economia e que fornece todos os nutrientes necessários ao bebê, foi notado que a prática de aleitamento materno foi pouca $\mathrm{e}$ por tempo insuficiente, optando as mães deste estudo pela alimentação artificial, que é mais onerosa.

O tempo médio em meses do uso de mamadeira foi 43,63 , sendo percebida a manutenção até 78 meses. Leite et al..$^{5}$ encontraram resultados semelhantes (tempo médio 40,6 meses e manutenção até 72 meses) e relataram que são várias as causas que se associam à opção pela forma mista ou artificial da aleitamento, como maior conveniência, experiências negativas já experimentadas com a amamentação e razões médicas; também observaram que cerca de $41 \%$ das mães tomam a decisão de não amamentar ainda durante a gestação.

Alguns estudos reportam o tempo mínimo de seis meses de aleitamento materno exclusivo para demonstrar uma associação negativa com a presença de hábitos deletérios, pois esse tempo é relatado na literatura como período suficiente para completo desenvolvimento morfofuncional do bebê $\hat{1}^{17,25,26}$. No entanto, Bezerra et al. ${ }^{21}$ mostram associação estatística significante entre o tempo de aleitamento materno recebido pela criança e hábitos deletérios e presença de hábitos com maloclusões.

A presença de hábitos de sucção não nutritivos foi observada em $176(53,3 \%)$ crianças e ausente em 154 (46,7\%), sendo que 70,45\% (124) das crianças que os apresentam não mamaram exclusivamente no peito até os seis meses de idade (Tabela 1). As relações entre estas duas variáveis foram estatisticamente significativas em nosso estudo. Dados semelhantes foram encontrados

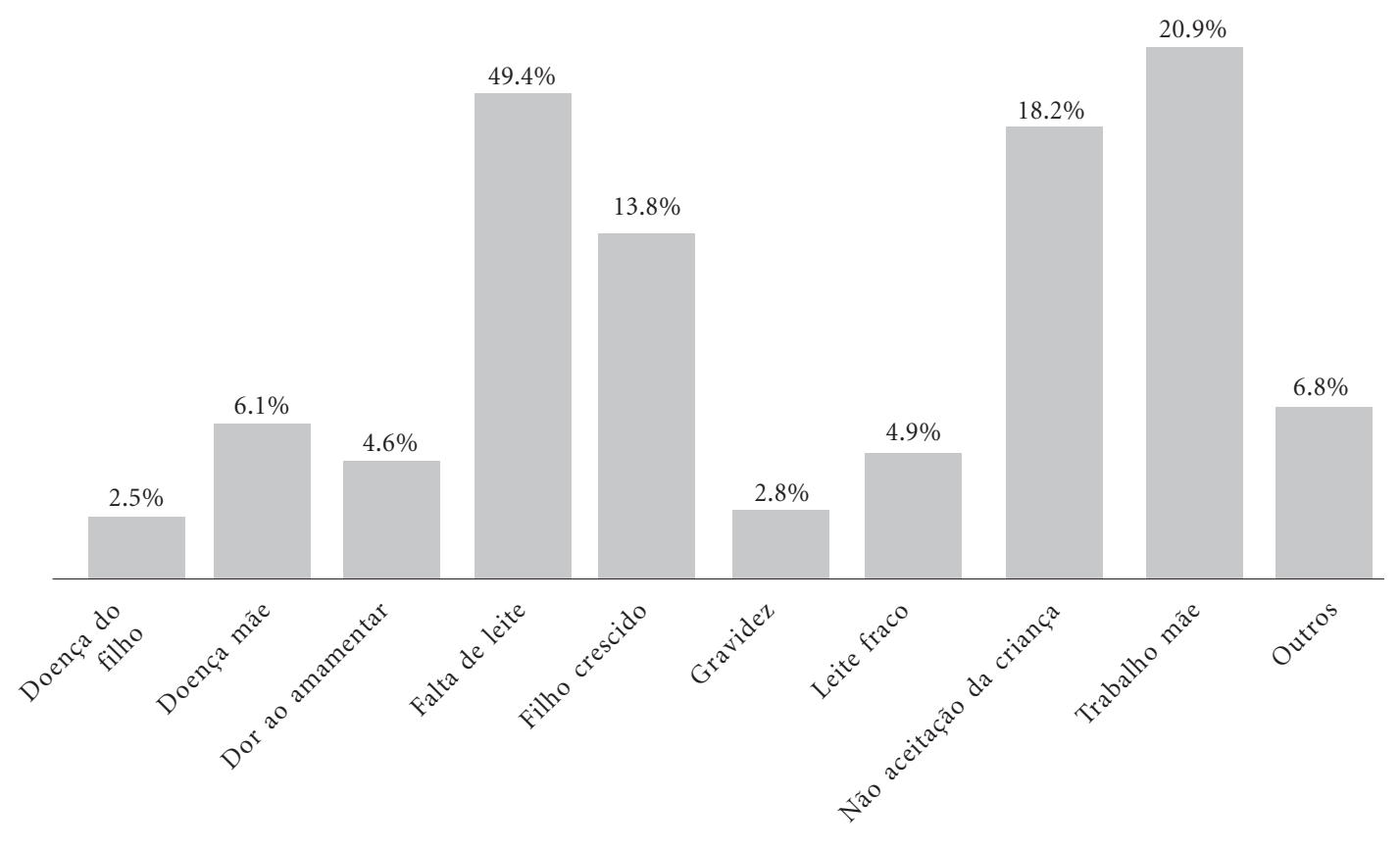

Gráfico 2. Distribuição percentual das 330 crianças segundo o principal motivo do desmame - Araçatuba (SP), 2008. 
nos estudos de Serra-Negra et al. ${ }^{25}$, Zuanon et al. ${ }^{15}$ e Sousa et al. ${ }^{16}$.

O hábito de chupar chupeta (Tabela 2) foi o mais frequente neste estudo, sendo encontrado em $44,4 \%$ das 232 crianças que apresentaram hábitos de sucção não nutritivos. Associando a presença do hábito de chupar chupeta com amamentação exclusiva e amamentação complementar, houve associação estatisticamente significante $\left(\mathrm{p}<0,0001\right.$ e $\left.\chi^{2}=36,65\right)$. No estudo de Bittencourt et. $a l$, o qual é um estudo transversal e retrospectivo semelhante ao nosso, o hábito de chupar chupeta foi o mais frequente $(55,6 \%)$, seguido pela onicofagia (10\%), pela sucção digital $(7,5 \%)$ e outros hábitos como morder objetos e ranger dentes. Esses achados mostram a baixa prevalência de sucção digital e alta frequência de sucção de chupetas, o que também é evidenciado em outros estudos ${ }^{16,25}$. As frequências variam de acordo com o tipo de estudo. Os estudos retrospectivos ou transversais apresentam maiores frequências de sucção digital quando comparados com os estudos longitudinais e prospectivos ${ }^{7}$.

A Tabela 3 demonstra que a prevalência de respiradores bucais percebida pelos pais ou responsáveis das crianças foi de 24,8\% (82). O hábito ocorria em $49 \%$ durante a noite; $49 \%$ durante o dia e a noite e $2 \%$ durante o dia. Houve associação estatisticamente significante entre a respiração bucal e a presença de aleitamento natural complementar ( $p=0,0395)$, ou seja, quanto maior o tempo de aleitamento natural, menores foram as chances de desenvolver a síndrome de respiração bucal.

Observando crianças de 2 a 11 anos, Leite et $a l .{ }^{5}$ verificaram que as que recebiam amamentação artificial apresentaram uma taxa $40 \%$ a mais de respiradores bucais. O aleitamento natural

Tabela 1. Distribuição numérica e percentual das 330 crianças estudadas de acordo com a presença de hábitos de sucção não nutritivos e período de tempo de amamentação materna exclusiva - Araçatuba (SP), 2008 .

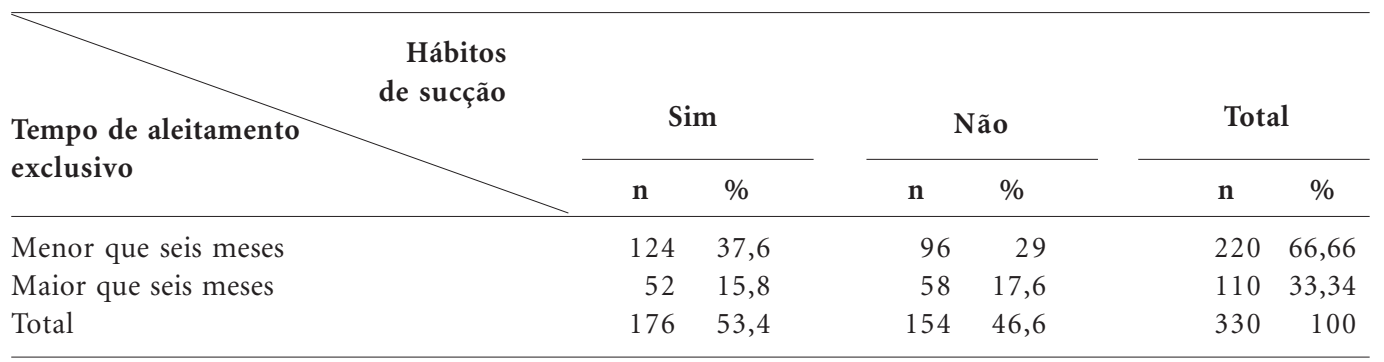

$\chi^{2}=19,23 ; \mathrm{p}<0,05$.

Tabela 2. Distribuição numérica das 330 crianças, de acordo com o tipo de hábitos de sucção não nutritivos, bem como sua duração em anos, em horas e período realizado - Araçatuba (SP), 2008.

\begin{tabular}{|c|c|c|c|c|c|c|c|c|c|c|c|c|c|}
\hline \multirow[b]{2}{*}{ Hábitos } & \multirow[b]{2}{*}{$\mathbf{n}$} & \multicolumn{3}{|c|}{ Duração em anos } & \multicolumn{5}{|c|}{ Duração em horas por dia } & \multicolumn{4}{|c|}{ Período } \\
\hline & & $\begin{array}{c}\text { Menos } \\
\text { que } 3 \\
\text { anos }\end{array}$ & $\begin{array}{l}\text { Mais } \\
\text { que } 3 \\
\text { anos }\end{array}$ & $\begin{array}{c}\text { Não } \\
\text { respondeu }\end{array}$ & $\begin{array}{l}0 \\
\frac{1-}{6}\end{array}$ & $\frac{6}{12}$ & $\frac{12}{18}$ & $\frac{18}{24}$ & $\begin{array}{c}\text { Não } \\
\text { respondeu }\end{array}$ & Dia & $\begin{array}{l}\mathrm{Dia} / \\
\text { noite }\end{array}$ & Noite & $\begin{array}{c}\text { Não } \\
\text { respondeu }\end{array}$ \\
\hline Chupar chupeta & 103 & 21 & 73 & 9 & 50 & 27 & 11 & 8 & 7 & 7 & 36 & 60 & 0 \\
\hline Chupar dedos & 22 & 5 & 14 & 3 & 10 & 4 & 1 & 3 & 4 & 10 & 4 & 8 & 0 \\
\hline Ranger dentes & 27 & 8 & 9 & 10 & 17 & 4 & 0 & 0 & 6 & 1 & 26 & 0 & 0 \\
\hline Roer unhas & 31 & 14 & 8 & 9 & 4 & 3 & 0 & 0 & 24 & 29 & 1 & 0 & 1 \\
\hline Roncar & 31 & 11 & 15 & 5 & 12 & 13 & 0 & 0 & 6 & 0 & 31 & 0 & 0 \\
\hline Babar à noite & 20 & 4 & 8 & 8 & 7 & 9 & 0 & 0 & 4 & 0 & 18 & 2 & 0 \\
\hline Outros & 6 & 1 & 1 & 4 & 2 & 1 & 0 & 0 & 3 & 3 & 1 & 1 & 1 \\
\hline Nenhum hábito & 154 & - & - & - & - & - & - & - & - & - & - & - & - \\
\hline
\end{tabular}

Obs.: algumas crianças apresentaram mais de um hábito. 
Tabela 3. Distribuição numérica e percentual das 330 crianças, de acordo com a presença de doenças na infância e a respiração bucal em relação ao recebimento ou não de aleitamento materno - Araçatuba (SP), 2008 .

\begin{tabular}{|c|c|c|c|c|c|c|c|}
\hline \multirow{2}{*}{ Variáveis } & \multicolumn{2}{|c|}{ Sim } & \multicolumn{2}{|c|}{ Não } & \multicolumn{2}{|c|}{ Total } & \multirow[b]{2}{*}{$\mathrm{p}$} \\
\hline & $\mathbf{n}$ & $\%$ & $\mathbf{n}$ & $\%$ & $\mathbf{n}$ & $\%$ & \\
\hline Respirador bucal & 82 & 24,85 & 248 & 75,15 & 330 & 100,00 & 0,0395 \\
\hline Doenças na infância & 147 & 44,55 & 183 & 55,45 & 330 & 100,00 & 0,0395 \\
\hline
\end{tabular}

permite uma tonicidade e postura correta da língua, com lábios em perfeito vedamento, propiciando o estabelecimento da respiração nasal, evitando a respiração bucal ${ }^{9,11,16}$.

Quanto à presença de doenças na infância, $44,5 \%$ (147) apresentavam, e elas eram ausentes em 55,5\% (183) (Tabela 3). As doenças respiratórias foram referidas por mais da metade pelas mães $(62,6 \%$ - 92) das crianças que tiveram doenças. Houve associação estatisticamente entre doenças da primeira infância (incluindo alergias e doenças respiratórias) e amamentação natural complementar $(\mathrm{p}=0,0395)$.

Neste estudo, foi constatada a associação entre a prática de amamentação materna exclusiva e a ausência de hábitos bucais não nutritivos, sendo de extrema importância na área da odontologia, principalmente pelas consequências que esses hábitos podem provocar na boca e na face. Vários estudos também evidenciaram essa relação $\mathrm{O}^{5,6,14,17,25}$.

A odontologia atualmente tem enfatizado a atenção primária de saúde, desde a vida intrauterina até a erupção dos dentes, podendo o profissional agir precocemente ${ }^{27}$ e assim alcançar melhores resultados em maior alcance e efetividade, pois os indivíduos absorvem melhor o conhecimento, evitando assim danos ao sistema estomatognático e prevenindo oclusopatias.
Considerando a importância do aleitamento materno na nutrição, diminuição da mortalidade infantil, melhoria dos aspectos psicológicos da criança e benefícios para a saúde bucal dos bebês, são extremamente importantes ações educativopreventivas com as gestantes incentivadas pelos profissionais de saúde, principalmente o cirurgião-dentista, qualificando assim a saúde bucal da gestante, bem como a saúde bucal de seu bebê.

\section{Considerações finais}

A prática do aleitamento foi verificada, porém por período de tempo inferior ao período considerado indispensável ao desenvolvimento normal do bebê. Existiu associação estatisticamente significante entre tempo e tipo de aleitamento e presença de hábitos de sucção não nutritivos.

O uso de chupeta esteve associado com o tempo de amamentação materna exclusiva e amamentação complementar, bem como o uso de mamadeiras com a amamentação complementar. O período de amamentação complementar também foi associado com a presença de respiração bucal e doenças na primeira infância.

Este estudo evidenciou a importância do incentivo do aleitamento materno como uma ação promotora de saúde bucal. 


\section{Colaboradores}

SAS Moimaz, NB Rocha, AJI Garbin e O Saliba participaram igualmente de todas as etapas da elaboração do artigo.

\section{Agradecimentos}

À Fundação de Amparo à Pesquisa do Estado de São Paulo pela bolsa de iniciação científica concedida.

\section{Referências}

1. Organização Mundial da Saúde. Evidências científicas dos dez passos para o sucesso no aleitamento materno. Brasília: Organização Pan-Americana da Saúde; 2001.

2. Brasil. Ministério da Saúde. Aleitamento materno. [documento na Internet]. 2005 [acessado 2005 maio 4]. Disponível em: http://portal.saude.gov.br/saude

3. Brasil. Ministério da Saúde. Secretaria de Políticas de Saúde. Estudos amostrais. Indicadores Dados Básicos. Indicadores de morbidade e fatores de risco. [documento na Internet] [acessado 2007 out 05]. 2007. Disponível em: http://tabnet.datasus.gov.br/cgi/ idb2004/d19.htm

4. Venancio SI, Monteiro CAA. A tendência da prática de amamentação no Brasil nas décadas de 70 e 80. Rev Bras Epidemiol 1998; 1:40-49.

5. Leite ICG, Rodrigues CC, Medeiros GV, Leite ICG, Pires LA. Associação entre aleitamento materno e hábitos de sucção não nutirtivos. Rev Ass Paul Cir Dent 1999; 53(2):151-155.

6. Baldrighi, SEZM, Pinzan A, Zwicker CVD, Michelini CR, Barros DR, Elias F. A importância do aleitamento natural na prevenção de alterações miofuncionais e ortodônticas. Rev Dent Press Ortod Ortop Facial 2001; 6(5):111-121.

7. Bittencourt LP, Modesto A, Bastos EPS. Influência do aleitamento sobre a frequência dos hábitos de sucção. Rev Bras Odontol 2001; 58(3):191-193.

8. Medeiros EB, Rodrigues MJ. A importância da amamentação natural para o desenvolvimento do sistema estomatognático do bebê. Rev Cons Reg Odontol Pernambuco 2001; 4(2):78-83.

9. Ferreira MIDT, Toledo OA. Relação entre tempo de aleitamento materno e hábitos bucais. Rev $A B O$ Nac 1997; 5(6):317-320.

10. Almeida GG, Spiri WC, Juliani CMCM, Paiva BSR. Proteção, promoção e apoio ao aleitamento materno em um hospital universitário. Cien Saude Colet 2008; 13(2):487-494.

11. Santos LM, Forte FDS, Bosco VL, Rocha MJC. Aleitamento materno e cárie dental. Rev Fac Odontol Univ Fed Bahia 2000; 20:34-37.

12. Longo GZ, Souza JMP, Souza SB, Szarfac SC. Crescimento de crianças até seis meses de idade, segundo categorias de aleitamento. Rev Bras Saúde Materno Infant 2005; 5(1):109-118.

13. Oliveira JF, Taveira MITA, Moreira GS, Zadorosny MV. Contribuição ao estudo do aleitamento materno: enfoque anticariogênico. Odontol Mod 1990; 17(9):6-9.

14. Queluz DP, Gimenez CMM. Aleitamento e hábitos deletérios relacionados à oclusão. Rev Paul Odon tol 2000; 17(6):16-20.

15. Zuanon ACC, Oliveira MF, Giro EMA, Maia JP. Influência da amamentação natural e artificial no desenvolvimento de hábitos bucais. J Brasil Odontop Odontol Bebê 2000; 2(8):303-306. 
16. Sousa FRN, Taveira GS, Almeida RVD, Padilha WWN. O aleitamento materno e sua relação com hábitos deletérios e maloclusão dentária. Pesq Bras Odontoped Clin João Pessoa 2004; 4(3):211-216.

17. Braghini M, Dolci GS, Ferreira EJB, Drehmer TM. Relação entre o aleitamento materno, hábito de sucção, forma do arco e profundidade do palato. Ortodontia Gaúcha 2001; 5(2):57-64.

18. Soares CAS, Totti JIS. Hábitos deletérios e suas conseqüências. Revista do CROMG 1996; 2(1):21-25.

19. Fonseca JS, Martins GA. Curso de estatística. São Paulo: Atlas; 1996.

20. Graphpad. Pacote estatístico - software. [programa de computador]. [acessado 2007 out 26]. Disponível em: http:/www.graphpad.com

21. Bezerra PKM, Cavalcanti AL, Bezerra PM, Moura C. Maloclusões, tipos de aleitamento e hábitos bucais deletérios em pré-escolares: um estudo de associação. Pesq Bras Odontop Clin Integr 2005; 5(3):267-274.

22. Organização Pan-Americana da Saúde (Opas). Guia alimentar para crianças menores de dois anos. Brasília: Ministério da Saúde, Secretaria de Políticas de Saúde, Organização Pan-Americana da Saúde; 2002.

23. World Health Organization (WHO). Global strategy for infant and young child feeding. Geneva: WHO; 2003.

24. Vasconcelos MGL, Lira PIC, Lima MC. Duração e fatores associados ao aleitamento materno em crianças menores de 24 meses de idade no estado de Pernambuco. Rev Bras Saúde Materno Infant 2006; 6(1):99-105.

25. Serra Negra JMC, Pordeus IA, Rocha JÚnior JF. Estudo da associação entre aleitamento, hábitos bucais e maloclusões. Rev Odontol Univ São Paulo 1997; 11(2):79-86.

26. Emmerich A, Fonseca L, Fonseca AM, Medeiros UV. Relação entre hábitos bucais, alterações orofaringianas e maloclusões em pré-escolares de Vitória, Espírito Santo, Brasil. Cad Saude Publ 2004; 20(3):689-697.

27. Bezerra LCA, Frias PG, Vidal SA, Macedo VC, Vanderlei LC. Aleitamento materno: avaliação da implantação do programa em unidades básicas de saúde do Recife, Pernambuco (2002). Cien Saude Colet 2007; 12(5):1309-1317.

Artigo apresentado em 13/08/2008

Aprovado em 09/02/2009

Versão final apresentada em 05/03/2009 